\title{
Unmet issues in transcatheter aortic valve implantation
}

Over the last 15 years, we have seen a rapid and exponential uptake of transcatheter aortic valve implantation (TAVI) for the treatment of patients with severe symptomatic aortic stenosis (1). This uptake has also been coupled with the evolution and advancement in transcatheter valve technologies. As such, a number of new TAVI devices have also been marketed, mainly with the aim of diminishing problems faced with first generation devices; notably, smaller delivery systems, less incidence of paravalvular leak (PVL) or need for permanent pacemaker implantation (PPI) and other device- or procedural related complications $(2,3)$.

In this focused issue of the journal, we provide a review series on unmet issues in the TAVI arena.

The peri-procedural risk assessment has been traditionally determined by the Society of Thoracic Surgeons' (STS) Predicted Risk of Operative Mortality, the Logistic European System for Cardiac Operative Risk Evaluation (EuroSCORE) I and more recently the EuroSCORE-II. The STS, logistic EuroSCORE-I and EuroSCORE-II scores have originally been conceived for cardiac surgery and though applied an further validated for TAVI, the major limitations are the lack of several comorbidities that are not captured $(4,5)$, yet considered important predictive risk factors such as frailty, porcelain aorta, hostile chest/radiation (4,6,7). Drs. Martin, Sperrin and Mamas provide an interesting overview regarding different models for assessing the pre-procedural risk of patients undergoing TAVI.

Pushing the envelope further, several reports have reported on the feasibility and safety of TAVI for the treatment of patients with bicuspid aortic valves. Drs. Patel and Leon discuss about this topic and the available data. In addition, Drs. Murdoch and Webb describe the literature around transcatheter valve-in-valve implantation for the treatment of degenerated surgical bioprostheses.

While doing the pre-TAVI workup, we have learned that the prevalence of coronary artery disease in patients undergoing TAVI ranges, depending of definitions, from $50 \%$ to $75 \%(8,9)$. Therefore, Drs. Perez, Thielhelm and Cohen discuss data around revascularizing patients with significant coronary artery disease and planned to undergo TAVI.

It has been shown that conscious sedation/local anesthesia is associated with shorter length of intensive care and overall hospital stay. In addition, conscious sedation/local anesthesia may favour an early discharge after TAVI, and the latter, without negatively affecting 30-day outcomes, including readmission rates (10). In this regard, Drs. Sato and Jones provocatively summarize the evidence around general anesthesia and sedation protocols for TAVI.

Newer-generation of TAVI devices comprise smaller delivery systems that have led to a significant reduction in access-site vascular complications but also, the increase use of full-percutaneous access. Hereof, Drs. Vora and Rao provide a review on percutaneous versus surgical cut-down for vascular access.

Once we are across the severely diseased aortic valve, the question comes to whether to implant the valve directly, without pre-implantation balloon aortic valvuloplasty or with valvuloplasty $(11,12)$. Dr. Kotronias and I overview the literature in this regard.

Cerebral embolic protection devices have made a lot of noise in our arena, mainly to reduce silent ischemic embolism; however, there is conflicting data with regards to hard end points such as clinically apparent cerebrovascular accidents or mortality (13). Thus, Dr. Nombela-Franco and colleagues update the current state of knowledge around this topic.

The need for PPI after TAVI $(14,15)$ is this still and unresolved matter. In their chapter, Drs. Toggweiler and Kobza review the "whys" of this issue.

Antithrombotic management after TAVI has become a frequent source of debate, mainly due to the incidence of clinical and subclinical valve thrombosis, and its subsequent risk for thromboembolic event and/or negative impact on valve durability. Dr. Mylotte's group discuss the prons and cons of antithrombotic agents after TAVI.

Long-term valve durability and the need for valve re-intervention $(16,17)$ is nowadays of a major importance, mostly with the trend to move toward lower-risk and younger patients. This topic is thoroughly covered by Drs. Kataruka and Otto.

Finally, Dr. Mylotte's group summarize a status update on TAVI.

I really believe that this special issue reflects a collaborative effort from multiple international colleagues and so, I hope that you enjoy it! 


\section{Acknowledgements}

None.

\section{References}

1. Bagur R, Webb JG. Standardising definitions for bioprosthetic structural valve deterioration and failure: the European avantgarde. EuroIntervention 2018;13:e1744-7.

2. Chu MW, Bagur R, Losenno KL, et al. Early clinical outcomes of a novel self-expanding transapical transcatheter aortic valve bioprosthesis. J Thorac Cardiovasc Surg 2017;153:810-8.

3. Bagur R, Teefy PJ, Kiaii B, et al. First North American experience with the transfemoral ACURATE-neo(TM) self-expanding transcatheter aortic bioprosthesis. Catheter Cardiovasc Interv 2017;90:130-8.

4. Bagur R, Rodes-Cabau J. Appropriate assessment of operative risk in patients with severe symptomatic aortic stenosis: importance for patient selection in the era of transcatheter aortic valve implantation. Ann Thorac Surg 2011;92:1157-8.

5. Bagur R, Martin GP, Nombela-Franco L, et al. Association of comorbid burden with clinical outcomes after transcatheter aortic valve implantation. Heart 2018. [Epub ahead of print].

6. Baumgartner H, Falk V, Bax JJ, et al. 2017 ESC/EACTS Guidelines for the management of valvular heart disease. Eur Heart J 2017;38:2739-91.

7. Rogers T, Koifman E, Patel N, et al. Society of Thoracic Surgeons Score Variance Results in Risk Reclassification of Patients Undergoing Transcatheter Aortic Valve Replacement. JAMA Cardiol 2017;2:455-6.

8. Kotronias RA, Kwok CS, George S, et al. Transcatheter Aortic Valve Implantation With or Without Percutaneous Coronary Artery Revascularization Strategy: A Systematic Review and Meta-Analysis. J Am Heart Assoc 2017;6.

9. Kotronias RA, Mamas MA, Bagur R. Revascularizing coronary artery disease in patients undergoing transcatheter aortic valve implantation. J Thorac Dis 2018;10:E79-82.

10. Kotronias RA, Teitelbaum M, Webb JG, et al. Early Versus Standard Discharge After Transcatheter Aortic Valve Replacement: A Systematic Review and Meta-Analysis. JACC Cardiovasc Interv 2018;11:1759-71.

11. Bagur R, Kwok CS, Nombela-Franco L, et al. Transcatheter Aortic Valve Implantation With or Without Preimplantation Balloon Aortic Valvuloplasty: A Systematic Review and Meta-Analysis. J Am Heart Assoc 2016;5.

12. Martin GP, Sperrin M, Bagur R, et al. Pre-Implantation Balloon Aortic Valvuloplasty and Clinical Outcomes Following Transcatheter Aortic Valve Implantation: A Propensity Score Analysis of the UK Registry. J Am Heart Assoc 2017;6.

13. Bagur R, Solo K, Alghofaili S, et al. Cerebral Embolic Protection Devices During Transcatheter Aortic Valve Implantation: Systematic Review and Meta-Analysis. Stroke 2017;48:1306-15.

14. Bagur R, Rodes-Cabau J, Gurvitch R, et al. Need for permanent pacemaker as a complication of transcatheter aortic valve implantation and surgical aortic valve replacement in elderly patients with severe aortic stenosis and similar baseline electrocardiographic findings. JACC Cardiovasc Interv 2012;5:540-51.

15. Bagur R, Choudhury T, Mamas MA. Transcatheter aortic valve implantation with the repositionable and fully retrievable Lotus Valve System(TM). J Thorac Dis 2017;9:2798-803.

16. Foroutan F, Guyatt GH, Otto CM, et al. Structural valve deterioration after transcatheter aortic valve implantation. Heart 2017;103:1899-905.

17. Bagur R, Pibarot P, Otto CM. Importance of the valve durability-life expectancy ratio in selection of a prosthetic aortic valve. Heart 2017;103:1756-9. 


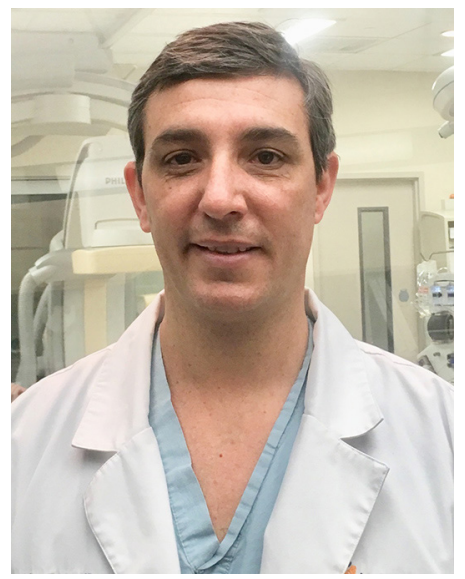

Rodrigo Bagur

\section{Rodrigo Bagur ${ }^{1,2}, \mathrm{MD}, \mathrm{PhD}$, FAHA}

${ }^{1}$ London Health Sciences Centre, London, Ontario, Canada;

${ }^{2}$ Keele Cardiovascular Research Group, Institute for Applied Clinical Science and Centre for Prognosis Research, Institute of Primary Care and Health Sciences, University of Keele, Stoke-on-Trent, UK.

(Email: rodrigobagur@yahoo.com) doi: $10.21037 /$ jtd.2018.10.13

Conflicts of Interest: The author has no conflicts of interest to declare. View this article at: http://dx.doi.org/10.21037/jtd.2018.10.13

Cite this article as: Bagur R. Unmet issues in transcatheter aortic valve implantation. J Thorac Dis 2018;10(Suppl 30):S3557-S3559. doi: 10.21037/jtd.2018.10.13 